\title{
Knee arthrodesis with the Ilizarov external fixator as treatment for septic failure of knee arthroplasty
}

\author{
M. Spina • G. Gualdrini • M. Fosco • \\ A. Giunti
}

Received: 29 July 2009/ Accepted: 28 March 2010/Published online: 28 April 2010

(c) The Author(s) 2010. This article is published with open access at Springerlink.com

\begin{abstract}
Background The authors report the results of femoraltibial fusion with an Ilizarov circular external fixator following septic loosening of knee prosthesis.

Materials and methods The series included 17 patients with a mean age of 62.9 years, treated from 1990 to 2007 with femoral-tibial fusion. The Cierny-Mader classification was used for clinical and anatomopathological evaluation; the Engh classification was used to assess the bone defect. Surgical treatment differed according to these criteria.

Results Healing was achieved in 13 out of 17 patients at the first surgical attempt in a mean time of 9.3 months. Mean follow-up was 30 months. Of the four complications, two patients had an intolerance to the external fixator that led to its early removal, and the other two had a septic intrarticular nonunion.

Conclusions The Ilizarov circular external fixator is a very reliable fixation system due to its low cost, versatility, stability under load, and low risk of septic dissemination. Nevertheless, an appropriate patient selection and a good surgeon's experience are necessary.
\end{abstract}

M. Spina · G. Gualdrini · M. Fosco - A. Giunti

VII Department of Orthopedic Surgery,

Rizzoli Orthopedic Institute, University of Bologna,

Bologna, Italy

\section{Spina $(\bowtie)$}

Prima Clinica di Ortopedia e Traumatologia,

Istituto Ortopedico Rizzoli, via Pupilli 1,

40136 Bologna, Italy

e-mail: spina.mauro@gmail.com
Keywords Septic prosthetic loosening . Knee arthrodesis - Rescue procedure . Ilizarov external fixator

\section{Introduction}

Femoral-tibial fusion remains one of the last treatment choices for recurrent septic failure of knee prostheses. It can be achieved by different surgical techniques, such as intramedullary nailing, mono/biaxial or circular external fixators, and fixation with long plates and screws.

In other studies, the rate of knee fusion following septic prosthetic loosening has been reported to range from $27 \%$ [1] up to $31-33 \%[2,3]$ and even $41-42 \%$ [4, 5]. However, authors often do not report the way that fusion is achieved; others use an unspecified external fixation with a success rate ranging from 67 to $90 \%$ [6-9], and with a mean fusion time that ranges from 4.4 to 6 months.

More precisely, other authors report that fusion rates with the Ilizarov circular external fixator range from 64$75 \%[10,11]$ to $83-93 \%[12,13]$ and even up to $100 \%$ [14]. Mean fusion times range from 6.8 to 13 months.

Among our series of 58 septic knee prostheses treated in our ward from 1990 to 2007, 17 (29.3\%) underwent femoral-tibial fusion. The fusions were attempted in all cases with the Ilizarov circular external fixator. The choice of fusion was dictated by bad local conditions of the knee (Fig. 1), the precarious general health status of the patient, and his determination to find a definitive solution to the problem. Another important factor was the number of failed prosthetic revision procedures due to septic loosening (Fig. 2).

The objective of our study was to evaluate the reliability of the Ilizarov circular external fixator as a surgical 


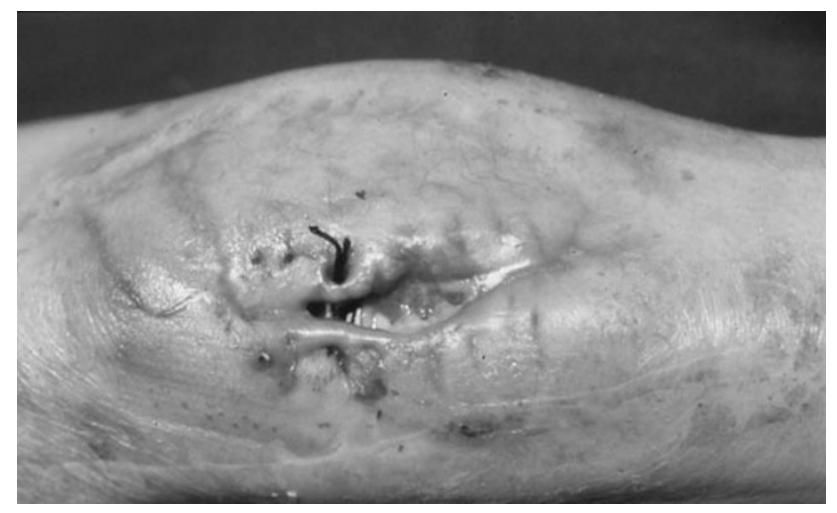

Fig. 1 Precarious local condition of the right knee in a septic prosthesis loosening

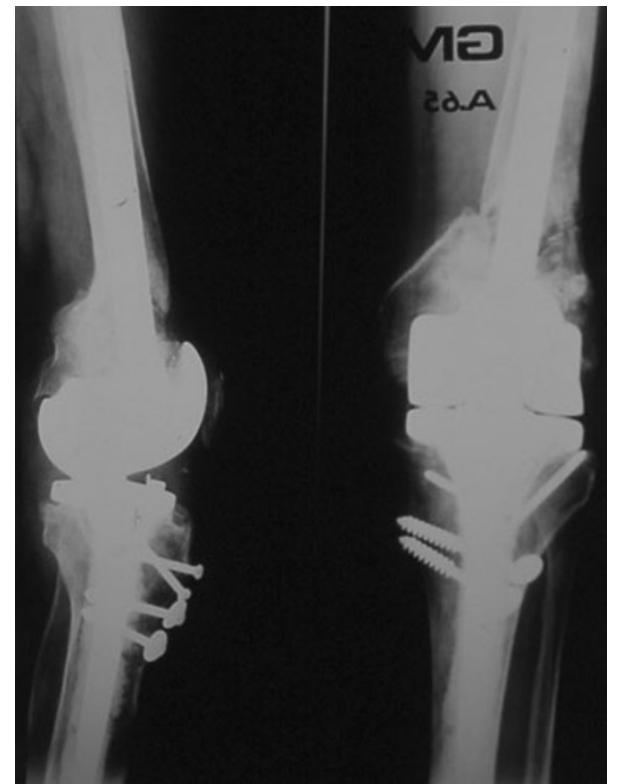

Fig. $2 \mathrm{~A} / \mathrm{P}$ and L X-ray of a septic knee revision prosthesis loosening technique for knee arthrodesis, and to compare it to other fusion procedures.

\section{Materials and methods}

The data for this investigation were collected and analyzed in compliance with the procedures and policies set forth by the Helsinki Declaration, and all patients gave their informed consent. The study was authorized by the local ethical committee.

The series included 17 femoral-tibial fusions, representing $29.3 \%$ of all septic knee prosthetic loosenings (58) treated on the First Ward of the Rizzoli Orthopedic Institute from 1990 to 2007 (Table 1). Nine patients were women (53\%) and eight were men (47\%); the mean age at the time of fusion was 62.9 years (women 68.3 and men
56.8), ranging from 26 to 80 years. Eleven patients had a secreting fistula. The microbiological culture examination was positive for Staphylococcus epidermidis in eight cases, Staphylococcus aureus in four cases, Enterococcus in four cases, and other bacterial species to lesser degrees (Fig. 3). The culture examination was negative in four patients (23.5\%), even when there were local conditions, and laboratory (ESR and CRP elevated) and radiological (locally increased uptake in total body scintigraphy with marked granulocytes) evaluations were positive for infection. Ten patients had previously been surgically treated for primary arthritis (58.8\%), four for posttraumatic arthritis (23.5\%), one for sequelae of tuberculous arthritis (5.9\%), one for rheumatic arthropathy (5.9\%), and one for arthropathy following pigmented villonodular synovitis $(5.9 \%)$. Five patients were treated at our institute from the implantation of the primary prosthesis, whereas the remaining 12 patients were initially treated at other institutes. The Cierny-Mader classification was used for clinical and anatomopathological assessment [15], while the Engh classification was used to evaluate bone defects [16]. According to the Cierny-Mader system, ten patients $(58.8 \%)$ belonged to group IV Bls, four patients $(23.6 \%)$ to group IV Bs, and the remaining patients (17.6\%) to group IV Bl.

According to the Engh classification, ten patients (58.8\%) were considered type II, and the remaining $(41.2 \%)$ were considered type III. Different surgical treatments were performed depending on the Engh classification.

For type II Engh patients the treatment involved:

- Injection of the fistulous tract, when present, with methylene blue dye;

- Removal of prosthetic components and cement mantle, samples taken for microbiological culture testing, than surgical debridement and regularization of the femoral and tibial bone surfaces;

- Femoral-tibial stabilization under compression with the Ilizarov external fixator, applying 5-6 $\mathrm{mm}$ diameter percutaneous half-pins with a hydroxyapatite coating for femoral arches, a distal femoral ring, and a pair of tibial rings stabilized with Kirschner wires;

- Specific or wide-ranging antibiotic therapy for four/six weeks.

For Engh type III patients (except in one case) the treatment involved:

- Injection of the fistulous tract, when present, with methylene blue dye

- Removal of prosthetic components cement mantle, samples taken for microbiological culture testing, than surgical debridement and regularization of the femoral and tibial bone surfaces 


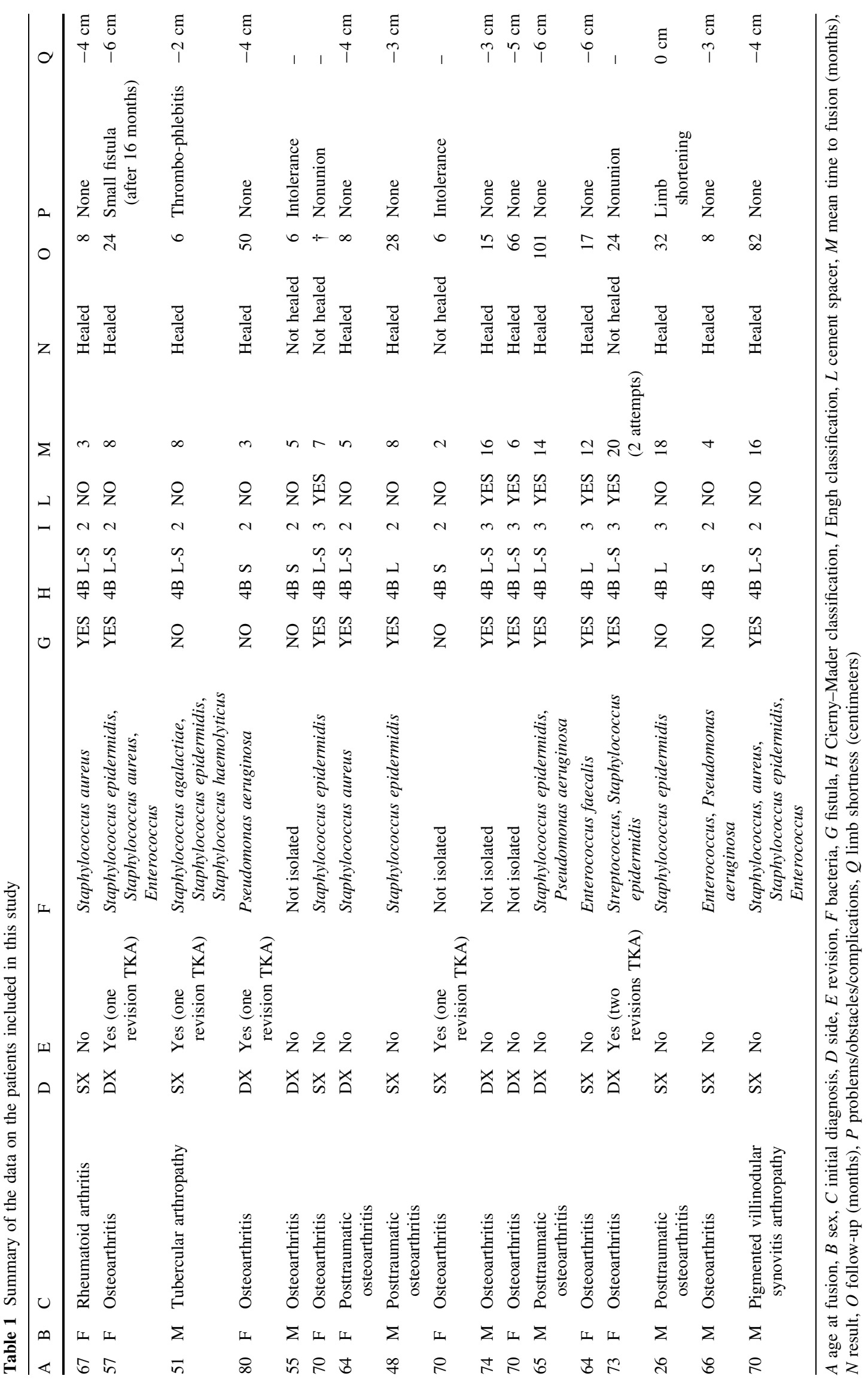



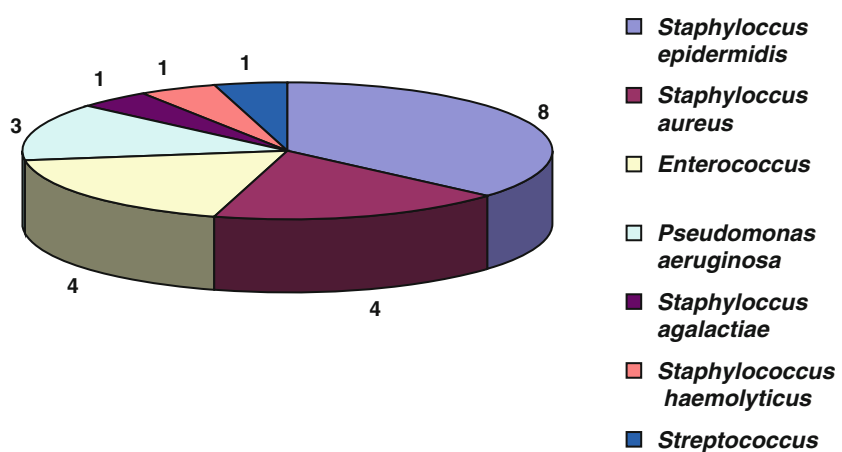

Fig. 3 Type and distribution of the isolated microbes

- Application of antibiotic-loaded cement spacer stained with methylene blue (Fig. 4)

- Hinged brace and specific or wide-ranging antibiotic therapy for four/six weeks

- Assessment of infection indices and clinical condition, then further surgical debridement followed by femoraltibial fusion with the external fixator

- Specific or wide-ranging antibiotic therapy for four/six weeks if the culture exam is positive at the time of fusion.

An additional surgical stage with the application of an antibiotic-loaded cement spacer is used for 4-6 weeks in patients belonging to Engh group III, because in our experience surgical debridement alone may not be sufficient to eradicate the infection in cases with large bone defects.

The type of antibiotic used in the spacer depended on the result of the microbiological test performed previously. In our series, in most cases we used vancomycin at a dose of 2-4 g of antibiotic per $40 \mathrm{~g}$ of cement.

In a 26-year-old patient with a final limb shortening of $11 \mathrm{~cm}$, femoral and tibial lengthening were performed at same time as the fusion.

Patients who were considered healed showed a continuous cancellous trabecular pattern from femur to tibia at

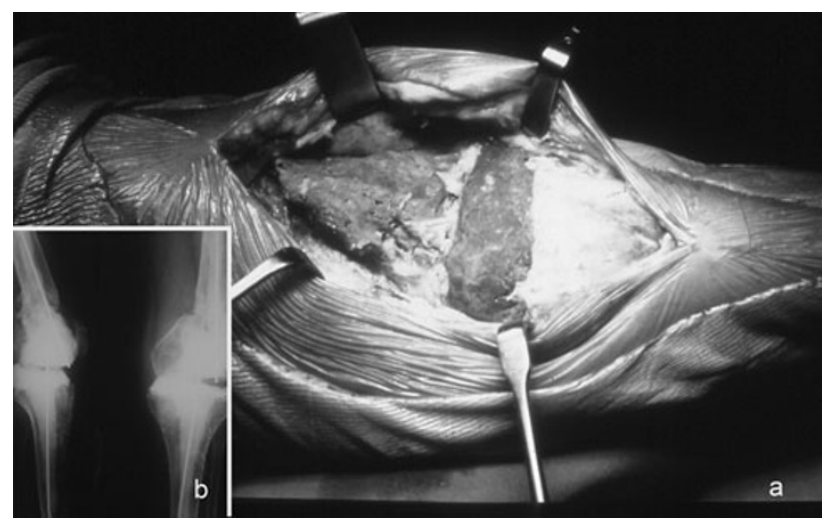

Fig. 4 a Antibiotic-loaded cement spacer stained with methylene blue following knee prosthesis removal. b A/P and L X-ray of a knee with cement spacer in situ

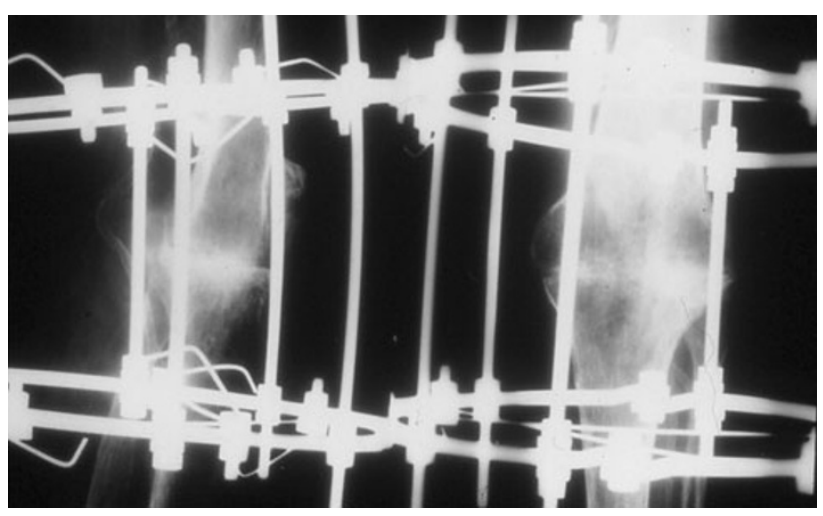

Fig. $5 \mathrm{~A} / \mathrm{P}$ and L X-ray showing continuity of the trabecularmedullary pattern in a patient treated with Ilizarov's external fixator

standard radiographs (Fig. 5) and no clinical and instrumental signs of an active infection. Stability at the fusion site was evaluated with the varus-valgus stress test. The femoral-tibial fusion was assessed both radiographically and clinically.

Technical notes for assembling the external fixator

\section{Femoral component}

The fixator is anchored to the femoral diaphysis by three or four 5-6-mm percutaneous half-pins coated in hydroxyapatite and fixed to two Ilizarov arches of the same diameter but different lengths (the distal one is longer). The arches are positioned perpendicular to the long axis of the femur. Four screws are recommended for patients over $60 \mathrm{~kg}$ in body weight. The distal arch is connected to a ring with a size proportional to the diameter of the knee and anchored to the distal femur by two Ilizarov wires.

\section{Tibial component}

The femoral distal ring is connected by three hinged rods to two rings of the same diameter that are anchored to the proximal tibial diaphysis by Ilizarov wires. For good fusion it is important that the fixator is connected to bone by Ilizarov wires on both sides of the subsequent fusion. In this setup the circular external fixator is bulky but effective. The hinged connection allows compression at the fusion site with deviations in flexion, external rotation and valgus of the tibia with respect to the femur. Proximal percutaneous screws and Ilizarov wires often cause local swelling of the skin. This is the most uncomfortable aspect for the patient (Figs. 6, 7).

\section{Results}

Among the 17 patients, 13 fusions were achieved at the first surgical attempt in a mean time of 9.3 months (range 
Fig. 6 Left knee in a patient treated by fusion using an Ilizarov circular external fixator. A splint is present to support the foot
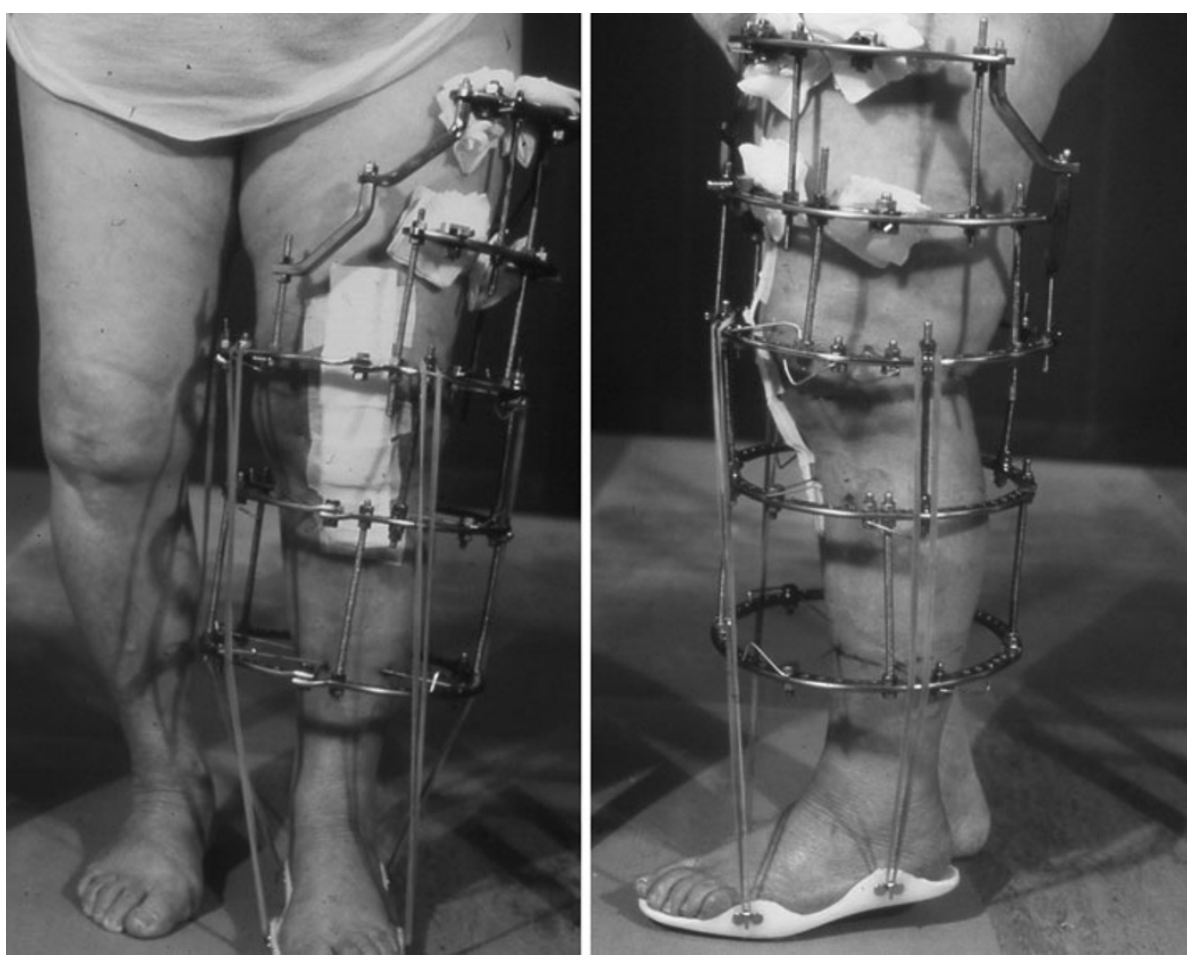

Fig. 7 Type IV Bls patient treated using an Ilizarov external fixator for left knee fusion. Functional stable limb bearing was achieved after 101 months
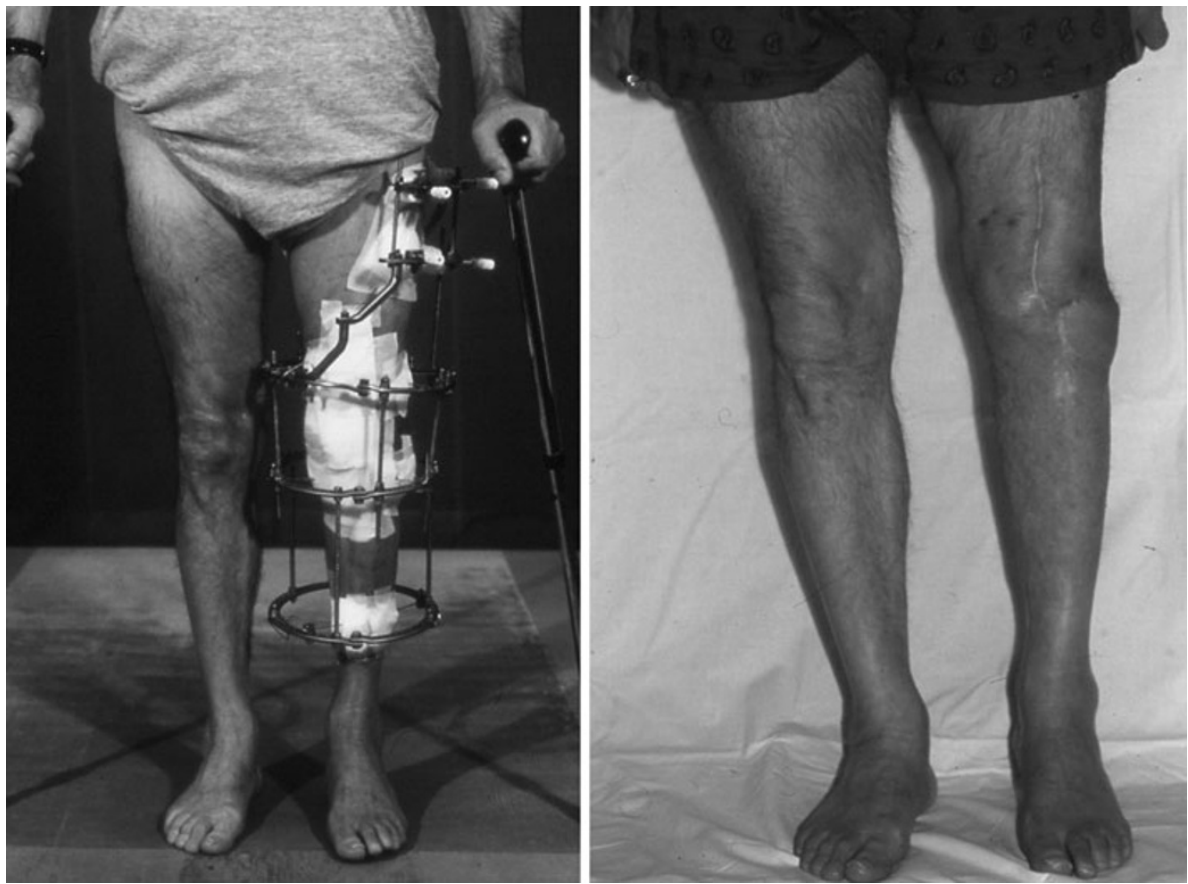

3-18 months) (Figs. 8, 9); the mean follow-up was 30 months (range 6-101 months) (Table 1).

To assess difficulties that occur during treatment with the Ilizarov external fixator, Paley's classification was used [17] to distinguish problems, obstacles and complications. Problems represent difficulties that require no operative intervention to resolve, while obstacles represent difficulties that require an operative intervention. All intraoperative injuries and all problems that are not resolved before the end of treatment are considered true complications. In our cohort there were four complications (23.5\%) that were responsible for treatment failure in four patients. Two patients belonging to Cierny-Mader IV Bls and the Engh type III group developed a septic intrarticular nonunion. The other two patients belonging to Cierny-Mader IV Bs and the Engh type II group developed an intolerance to the external fixator that led to its early removal. In all four patients, further attempts at fusion with other surgical techniques were abandoned and 


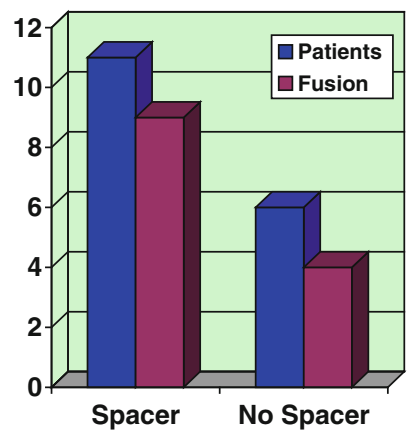

Fig. 8 Comparison of patients according to treatment and fusion rate

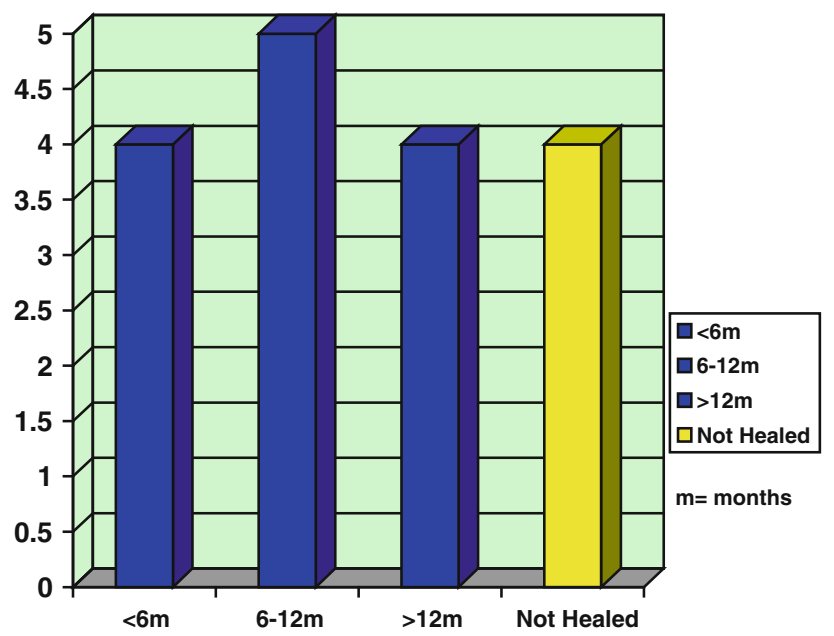

Fig. 9 Duration of treatment with Ilizarov fixator in healed patients

a hinged brace was applied. The mean residual limb length discrepancy was $3.8 \mathrm{~cm}$ (range $0-6 \mathrm{~cm}$ ). One obstacle occurred in a 26-year-old patient with a final limb shortening of $11 \mathrm{~cm}$; femoral-tibial fusion and femoral and tibial lengthening using the Ilizarov technique were performed at the same time, thus restoring the original length of the limb.

Problems included a thrombophlebitis in a patient with severe venous insufficiency of the lower limbs and previous deep venous thrombosis; this was treated with pharmacological therapy. Another was a small fistula at a surgical wound that developed in another patient 16 months after the removal of the external fixator, which resolved after specific antibiotic therapy. Among other problems, there were superficial wound infections of percutaneous screws and Ilizarov wire tracts that were never quantified but which always resolved with local disinfection.

\section{Discussion}

Femoral-tibial fusion is a valid alternative upon septic failure of primary and revision total knee arthroplasty, and is usually well tolerated by patients.
This treatment restores good limb loading, decreasing pain and eliminating infection.

Circular external fixation provides stability at the fusion site and correct femoral-tibial alignment in flexion, external rotation and valgus deviation. Any type of correction is possible without the need to take the patient into the operating room. The circular fixator provides very good stability, so daily load-bearing may be allowed without limitation. It is a low-cost option from a hospital economics perspective, and it ensures a low risk of infection. In particular cases during treatment for femoral-tibial fusion, the fixator can restore severe limb-length discrepancy by applying a distraction osteogenesis technique on the tibial and/or femoral side.

Disadvantages of circular external fixator include:

- A long learning curve

- Objective discomfort for the patient due to the wide field of the device

- Nonrigid fixation due to flexible metal wires and percutaneous screws

- Cutaneous infections frequently occur at wire entry sites

- Loosening and breakage of percutaneous screws

- Long treatment times.

Knee arthrodesis achieved by various types of intramedullary nailing has a success rate ranging from 67 to $100 \%[6,18-24]$ in a mean time of about six months. Using intramedullary nailing in a knee with a periprosthetic infection poses a number of problems, such as the risk of spreading the infection into the medullary canal, the difficulty involved in treating infection recurrence, the possibility of nail migration or breakage, and the impossibility of performing a compression at the fusion site and clinically assessing its stability during treatment [23, 25].

The technique of femoral-tibial fusion with a monoaxial or biaxial external fixator has a success rate that ranges from 68 [26] to $89 \%$ [27] and up to $100 \%$ [28, 29]. These fixators, especially monoaxial ones, are fairly well tolerated by patients. However, they do not allow significant changes in the axis, and, due to their structural characteristics, they are rigid and not entirely reliable for complete load-bearing [30].

Finally, bone fusion with dual compression plates has a success rate ranging from 80 [31] to $100 \%$ [32]. The authors, however, report high rates of complications (18.2\%) such as stress fractures and persistent infection. Healing in some cases was achieved after repeated surgical attempts. Bone fixation with plates and screws is rigid and enables axial compression [33]. It is, however, a complex procedure that is very invasive and at risk of infection. Nichols et al. [32] advise against this technique in the presence of widespread infection. 


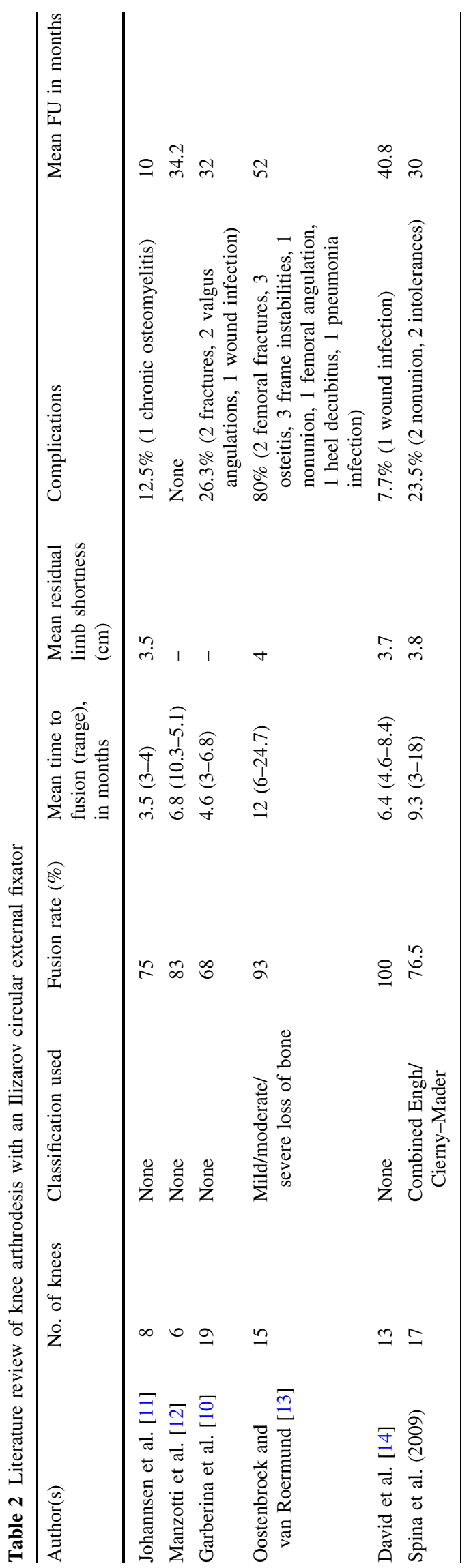

The existing literature on femoral-tibial fusion with an Ilizarov circular external fixator reports success rates that vary in different studies from 64 [10] to 100\% [14] (Table 2). In our series, the rate of complete healing was $76.5 \%$ at the first surgical attempt in a mean time of 9.3 months. Failures consisted of four patients $(23.5 \%$ of the entire group); two of these patients had bad general health conditions; one patient died a few months after removal of the external fixator; another did not heal despite a second attempt at fusion with an Ilizarov external fixator. The other two patients were affected by an anxiousdepressive syndrome that contributed to severe intolerance to the external fixator, so its early removal was inevitable. This event accounted for $50 \%$ of the failures, so we believe in the importance of carefully assessing the patient's ability to cooperate before treatment.

Our selection of an Ilizarov circular external fixator was dictated by its low cost, versatility, stability under load, possibility to performing modifications during treatment, and low risk of septic dissemination. Nevertheless, in our opinion, careful patient selection is required, as old age and psychological intolerance are generally compromising factors. The treatment time is long and an experienced surgeon is needed to assemble the external fixator and manage it later.

\section{Conflict of interest None.}

Open Access This article is distributed under the terms of the Creative Commons Attribution Noncommercial License which permits any noncommercial use, distribution, and reproduction in any medium, provided the original author(s) and source are credited.

\section{References}

1. Husted H, Toftgaard Jensen T (2002) Clinical outcome after treatment of infected primary total knee arthroplasty. Acta Orthop Belg 68(5):500-507

2. Blom AW, Brown J, Taylor AH, Pattison G, Whitehouse S, Bannister GC (2004) Infection after total knee arthroplasty. J Bone Joint Surg Br 86(5):688-691

3. Weng X, Li L, Qui G, Li J, Tian Y, Hen J, Wang Y, Jin J, Ye Q, Zhao H (2002) Treatment of infected total knee arthroplasty. Zhonghua Wai Ke Za Zhi 40(9):669-672

4. Bengston S, Knutson K, Lidgren L (1989) Treatment of infected knee arthroplasty. Clin Orthop Relat Res 245:173-178

5. Hanssen AD, Trousdale RT, Osmon DR (1995) Patient outcome with reinfection following reimplantation for the infected total knee arthroplasty. Clin Orthop Relat Res 321:55-67

6. Mabry TM, Jacofsky DJ, Haidukewych GJ, Hanssen AD (2007) Comparison of intramedullary nailing and external fixation knee arthrodesis for the infected knee replacement. Clin Orthop Relat Res 464:11-15

7. Figgie HE III, Brody GA, Inglis AE, Sculco TP, Goldberg VM, Figgie MP (1987) Knee arthrodesis following total knee arthroplasty in rheumatoid arthritis. Clin Orthop 224:237-243 
8. Klinger HM, Spahn G, Schultz W, Baums MH (2006) Arthrodesis of the knee after failed infected total knee arthroplasty. Knee Surg Sports Traumatol Arthrosc 14(5):447-453

9. Rudolph F, Fengler F, Hein W (1989) Arthrodesis as an alternative in infected knee arthroplasty. Beitr Orthop Traumatol 36(8):374-380

10. Garberina MJ, Fitch RD, Hoffmann ED, Hardaker WT, Vail TP, Scully SP (2001) Knee arthrodesis with circular external fixation. Clin Orthop 382:168-178

11. Johannsen HG, Skov O, Weeth ER (2005) Knee arthrodesis with external fixator after infected knee arthroplasty. Ugeskr Laeger 167(35):3295-3296

12. Manzotti A, Pullen C, Deromedis B, Catagni MA (2001) Knee arthrodesis after infected total knee arthroplasty using the Ilizarov method. Clin Orthop Relat Res 389:143-149

13. Oostenbroek HJ, van Roermund PM (2001) Arthrodesis of the knee after an infected arthroplasty using the Ilizarov method. J Bone Joint Surg Br 83:50-54

14. David R, Shtarker H, Horesh Z, Tsur A, Soudry M (2001) Arthrodesis with Ilizarov device for failed knee arthroplasty. Orthopedics 24:33-36

15. Cierny G, Mader JT, Penninck JJ (1985) A clinical staging system for adult osteomyelitis. Contemp Orthop 10(5)

16. Engh GA, Ammeen DJ (1999) Bone loss with revision total knee arthroplasty: defect classification and alternatives for reconstruction. AAOS Instr Course Lect 48(22):167-175

17. Paley D (1990) Problems, obstacles, and complications of limb lengthening by the Ilizarov technique. Clin Orthop Relat Res 250:81-104

18. Wilde AH, Stearns KL (1989) Intramedullary fixation for arthrodesis of the knee after infected total knee arthroplasty. Clin Orthop Relat Res 248:87-92

19. Bargiotas K, Wohlrab D, Sewecke JJ, Lavinge G, DeMeo PJ, Sotereanos GN (2006) Arthrodesis of the knee with a long intramedullary nail following the failure of a total knee arthroplasty as the result of infection. Surgical technique. J Bone Joint Surg Am 89:103-110

20. Senior CJ, da Assuncao RE, Barlow IW (2007) Knee arthrodesis for limb salvage with an intramedullary couplet nail. Arch Orthop Trauma Surg 128:683-687
21. Volpi R, Dehoux E, Touchard P, Mensa C, Segal P (2004) Knee arthrodesis using a customized intramedullary nail: 14 cases. Rev Chir Orthop Reparatrice Appar Mot 90(1):58-64

22. Lai KA, Shen WJ, Yang CY (1998) Arthrodesis with a short Huckstep nail as a salvage procedure for failed total knee arthroplasty. J Bone Joint Surg 80(3):380-388

23. Waldman BJ, Mont MA, Payman KR, Freiberg AA, Windsor RE, Sculco TP, Hungerford DS (1999) Infected knee arthroplasty treated with arthrodesis using a modular nail. Clin Orthop Relat Res 367:230-237

24. Jorgensen PS, Torholm C (1995) Arthrodesis after infected knee arthroplasty using long arthrodesis nail. A report of five cases. Am J Knee Surg 8(3):110-113

25. Donley BG, Matthews LS, Kaufer H (1991) Arthrodesis of the knee with an intramedullary nail. J Bone Joint Surg Am 73(6):907-913

26. Rand J, Bryan R, Chao E (1987) Failed total knee arthroplasty treated by arthrodesis of the knee using the Ace-Fisher apparatus. J Bone J Surg 69:39

27. Parratte S, Madougou S, Villaba M, Stein A, Rochwerger A, Curvale G (2007) Knee arthrodesis with a double mono-bar external fixators to salvage infected knee arthroplasty: retrospective analysis of 18 knees with mean seven-year follow-up. Rev Chir Orthop Reparatrice Appar Mot 93(4):373-380

28. Fidler MW (1983) Knee arthrodesis following prosthesis removal: use of the Wagner apparatus. J Bone Joint Surg 65B:29-31

29. Wade PJ, Denham RA (1984) Arthrodesis of the knee after failed knee replacement. J Bone Joint Surg Br 66:362-366

30. Hak DJ, Lieberman JR, Finerman GA (1995) Single plane and biplane external fixators for knee arthrodesis. Clin Orthop Relat Res 316:134-144

31. Munzinger U, Knessl J, Gschwend N (1987) Arthrodesis following knee arthroplasty. Orthopade 16(4):301-309

32. Nichols SJ, Landon GC, Tullos HS (1991) Arthrodesis with dual plates after failed total knee arthroplasty. J Bone Joint Surg Am 73(7):1020-1024

33. Christie MJ, De Boer DK, McQueen DA, Cooke FW et al (2003) Salvage procedures for failed total knee arthroplasty. J Bone J Surg Am 85:S58-S62 